\title{
A novel 11 p13 microdeletion encompassing PAX6 in a Chinese Han family with aniridia, ptosis and mental retardation
}

Ping $\mathrm{Hu}^{1 \dagger}$, Lulu Meng ${ }^{1 \dagger}$, Dingyuan Ma ${ }^{1}$, Fengchang Qiao ${ }^{1}$, Yan Wang ${ }^{1}$, Jing Zhou ${ }^{1}$, Long $\mathrm{Yi}^{2}$ and Zhengfeng $\mathrm{Xu}^{{ }^{1 *}}$

\begin{abstract}
Purpose: To explore possible genetic aberrations in a Chinese family with aniridia, ptosis and mental retardation, and provide genetic evidence for the prenatal diagnosis.

Methods: 14 exons of PAX6 in the proband were sequenced by the Sanger sequencing technique. Multiplex ligation-dependent probe amplification (MLPA) technique was employed to further explore gene alterations of PAX6. Single nucleotide polymorphisms-array (SNP-array) assay was applied to screen potential pathologic genome-wide copy number variations (CNV).

Results: There were no detectable pathogenic mutations in the 14 exons of PAX6 in the proband. MLPA indicated a heterozygous deletion encompassing all PAX6 gene regions covered and a partial upstream region. SNP-array assay detected a heterozygous 11 p13 microdeletion with a length of $518 \mathrm{~kb}$ in the proband, spanning two whole annotated genes, elongation factor protein 4 (ELP4), the paired box gene 6 (PAX6), and partial IMP1 inner-mitochondrial membrane (IMMPIL) gene. SNP-array revealed her affected brother carried the identical deletion.
\end{abstract}

Conclusions: The $518 \mathrm{~kb}$ heterozygous deletion in 11p13 encompassing PAX6 should be the genetic etiology for the familial aniridia.

Keywords: PAX6, 11 p13 deletion, Mental retardation, Aniridia, SNP-array

\section{Background}

Congenital aniridia (OMIM 106210) is a kind of eye disorder characterized by complete or partial hypoplasia of the iris. The worldwide prevalence of aniridia was estimated to be 1:50 000 to 1:100 000 in the year of 2013 [1]. About two-thirds of reported aniridia cases were familial and showed a dominant inheritance manner with nearly complete penetrance, the remaining one-third were sporadic [2]. PAX6 (OMIM 607108), a well-known aniridia disease-causing gene located in chromosome $11 \mathrm{p} 13$ region, contains 14 exons and encodes a protein of 422 amino acids. As a transcriptional factor, PAX6 is involved in the development of a diversity of tissues and organs

\footnotetext{
*Correspondence: njxzf@126.com

${ }^{\dagger}$ Equal contributors

'State key Laboratory of Reproductive Medicine, Department of Prenatal Diagnosis, Nanjing Maternity and Child Health Care Hospital Affiliated to Nanjing Medical University, 123\# Tianfei Street, Baixia District, Nanjing 210029, China

Full list of author information is available at the end of the article
}

including ocular tissues, olfactory bulb, neural tube, gut and pancreas $[3,4]$. Heterozygous loss of function of PAX6 was identified in about $90 \%$ aniridia cases, with intragenic mutations accounting for approximately two-thirds of cases and chromosomal rearrangements accounting for the other one-third [2].

Previous studies demonstrated that people harboring PAX6 mutation displayed a clinical symptom spectrum including aniridia, corneal opacification, keratitis, cataract, glaucoma, lens dislocation, ciliary body hypoplasia, foveal hypoplasia, strabismus, nystagmus, Peter's anomaly, optic nerve defects [5], hyposmia, abnormal inter-hemispheric auditory transfer [6], impaired islet function [7] and brain structure abnormalities [8]. Until now, approximately 357 intragenic mutations have been documented in the PAX6 mutation database (http://www.hgu.mrc.ac.uk/Softdata/ PAX6/), while microdeletion cases associated with PAX6 region were rare.




In this study, we report on a novel genomic microdeletion including PAX6 in a small Chinese family with aniridia, cataract, ptosis and mental retardation detected by SNParray assay.

\section{Case presentation}

This three-generation family included four affected patients, two of them were available for this study (Figure 1A).

The proband (II:1), a 31-years-old pregnant Chinese Han female who suffered from congenital bilateral complete absence of iris, cataracts, bilateral ptosis was referred to our center for genetic counseling at 7 weeks' gestation. She is the first child born to a non-consanguineous couple. Her father is healthy while her mother (I:1) and bother were suffered by anirida. She is normal in growth and speaking during her development. She did not display any intellectual disability. Her first child, who was also affected by anirida, was born when she was 24 . Her mother and first child deceased at the age of 45 and 5 due to non-medically related causes. Serum growth hormone $(\mathrm{GH})$, thyroid stimulating hormone (TSH), free triiodothyronine (fT3),

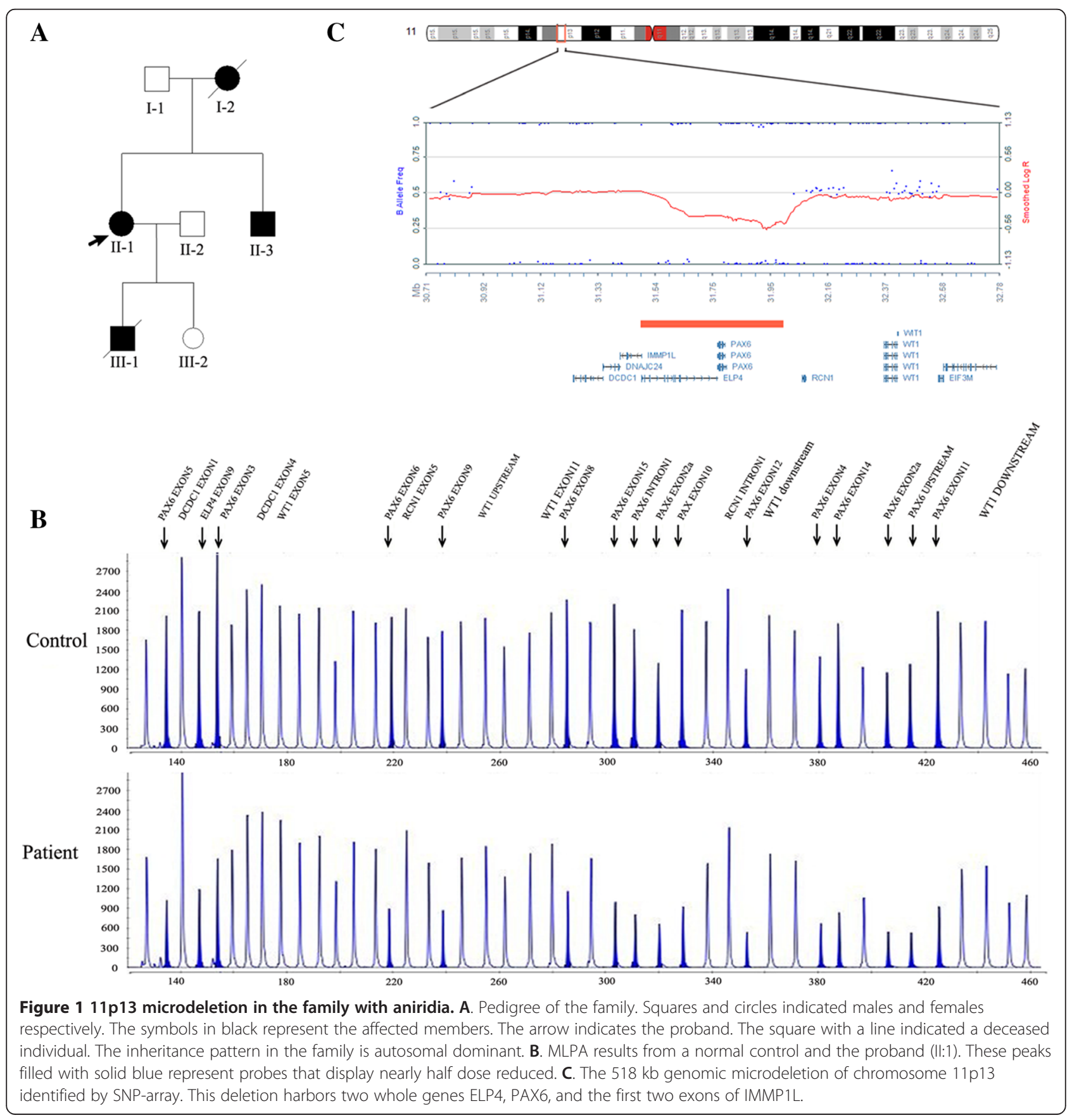


and free thyroxineIndex (fT4) levels were all in normal range. Ultrasonography results indicated her internal organs were normal.

Her affected brother (II:2) of 26-years-old was also recruited for clinical tests. He is normal in height and weight. Physical examination was normal, while minor anomalies including prominent forehead, short philtrum were observed. He was diagnosed with mental retardation when he was 7 years-old. His IQ was 70 when he was 17 years-old. Ultrasonography results indicated his internal organs were normal. MRI did not reveal any structure abnormalty.

\section{Results}

\section{Mutation analysis for PAX6}

Mutation screening of 14 PAX6 exons by Sanger sequencing did not reveal any abnormality in the proband (data not shown).

\section{Multiple ligation-dependent probe amplification (MLPA) assays}

MLPA assays were used to identify possible PAX6 microdeletion (Figure 1B). MLPA results revealed that the height of all these probes in the ELP4 and PAX6 displayed nearly half-dose reduced, whereas probes targeting genes up- or downstream to the deletion such as DCDC1, $R C N 1, W T 1$, exhibited nearly the same height as the normal. MLPA assay on amniotic fluid cells collected at the 18 gestation week revealed the fetus (III:2) did not inherit the deletion from the mother. The female baby was born at 39 weeks, and physical examinations confirmed no abnormalities especially in the eyes. Due to the unavailability of the DNA samples, we are unable to determine whether the deletion existed in patients (I:2, III:1).

\section{SNP-array assay}

SNP-array test was applied to find possible pathologic copy number variations besides PAX6 microdeletion in the proband and her brother. The results identified a heterozygous deletion in the $11 \mathrm{p} 13$ region expanding from $31,486,211$ to $32,004,859$ bp (hg18) with a length of $518 \mathrm{~Kb}$ in the proband. The deleted genomic fragment contains two whole genes, elongation factor protein 4 (ELP4), the paired box gene 6 (PAX6), and partial IMP1 inner-mitochondrial membrane (IMMP1L) gene (exon1, 2). Additionally, an identical 11p13 deletion was identified in proband's brother (Figure 1C). However, no other pathologic copy number variations were found in the genomewide SNP-array scanning in both of them.

\section{Discussion}

In this study, we characterized a novel $518 \mathrm{~kb}$ deletion on 11 p13 in a three-generation Chinese Han family with familial aniridia. In addition to the aniridia and cataracts, interestingly, one of them also presented with mental retardation. We speculated that PAX6, which lies in the deleted region, primarily accounted for these clinical features.

Neurological disorders such as mental retardation [9], autism [10], epilepsy, cognitive impairments or behavioral abnormalities [11] are uncommon in individuals with PAX6 mutations. Chromosome microdeletions were much less commonly reported in aniridia patients than PAX6 point mutations. As far as we know, only 14 cases were found to carry genomic microdeletion encompassing PAX6 or its regulatory region but not WT1 to date [12-20], and only one of them displayed neurodevelopmental disorders. Davis LK et al. reported a 13-year-old patient with aniridia, autism, and mental retardation carrying a $1.3 \mathrm{Mb}$ heterozygous microdeletion, approximately $35 \mathrm{~kb}$ distal to the last exon of PAX6 [15]. Further study indicated the deletion was inherited from the mother who presented with aniridia, depression, anxiety, and social awkwardness. Though the copy number of PAX6 was intact in the family, the regulatory genomic region of $P A X 6$ nearby ELP4 included in the deletion was believed to be the etiology. In our study, a $518 \mathrm{~kb}$ submicroscopic deletion including PAX6, ELP4 and partial IMMP1L was identified. All the four patients in the family were normal in other physical development, but the proband's brother presented with moderate mental retardation, indicating a marked intra-familial variation.

Neurological disorders presented in individuals with PAX6 mutations can be explained by the PAX6 expression pattern and outcome from animal model research. PAX6 expressed in the telencephalon, diencephalon, caudal rhombencephalon, myelencephalon and spinal cord, but not mesencephalon in the embryonic period, and adult brains [21-23], suggesting a role in the brain development. Pax6 mutant mice/rats displayed abnormal development in arealisation of the cerebral cortex, formation of the prosencephalon-mesencephalon boundary, axon guidance, differentiation of neurons from glia and neuronal migration in the cerebellum [24]. Brain defects were occasionally found to be related with PAX6 mutations. Magnetic resonance imaging (MRI) examination on patients with PAX6 mutations revealed a spectrum of brain abnormalities including absence/hypoplasia of the anterior commissure, reduced olfaction [25], polymicrogyria, absence of pineal gland [8], reduction in the white matter in the corpus callosum and grey matter in the anterior cingulated cortex, cerebellum, medial temporal lobe [26]. However, the patients' brains were normal detected by brain MRI test in our case. The detailed molecular mechanisms underlying the mental retardation and brain structure defects of PAX6 mutation is yet unknown.

Congenital aniridia can also occur in the WAGR syndrome (Wilms' tumor, aniridia, genitourinary abnormalities, 
and mental retardation), a syndrome due to a contiguous gene deletion encompassing both PAX6 and WT1. Individuals, particularly children under 6 years old, with WT1 deletion may predispose to Wilms' tumor, a kidney tumor with childhood onset [13]. Defining the accurate breakpoints of the 11p13 deletion may contribute to the genetic counseling, disease prognosis and prevention [1].

Another interesting gene is EPL4, which encodes a component of the six subunit elongator complex, a histone acetyltransferase complex that associates directly with the RNA polymerase II (Pol II) holoenzyme involved in transcriptional elongation. DNaseI hypersensitivity mapping and reporter transgenic assays revealed the presence of several putative cis-regulatory elements, including ones driving expression in lens and retina [27]. These elements reside within introns of the adjacent, ubiquitously expressed ELP4 gene, but are nevertheless thought to be PAX6-specific long-range control elements [28]. Andrea $\mathrm{C}$ et al. demonstrated that three SNPs in ELP4 affect the size of the optic nerve head [29]. Zhang X et al. revealed that a 3 ' deletion to the PAX6 gene including the ELP4 was identified in one three-generation family with total aniridia [19]. A part of IMMP1L gene was also deleted in our patients. IMMP1L encode a subunit of IMP (mitochondrial inner membrane peptidase) complex which plays a role in the generating mature, active proteins in the mitochondrial intermembrane space by proteolytically removing the mitochondrial targeting presequence of nuclear-encoded proteins.

In this study, we accurately identified the pathologic genetic aberration of the family using SNP-array. In fact, consistent with our prenatal genetic diagnosis assistance, they delivered a healthy baby. The couple was informed they have a $50 \%$ chance to birth a healthy baby in subsequent pregnancies.

\section{Conclusions}

In summary, this study identified a novel deletion containing PAX6 in a Chinese family, expanding the mutation spectrum of PAX6 aberration. The $518 \mathrm{~kb}$ heterozygous deletion of chromosome 11p13 may be the cause of the familial aniridia, congenital ptosis and slight mental retardation in this family. The function of the deleted genes are needed to be further studied. This work emphasizes the necessity to screen for possible microdeletion related with PAX6 in patients with aniridia particularly when result of conventional sequencing analysis for PAX6 is negative.

\section{Methods}

\section{DNA extraction and sequencing}

Genomic DNA was extracted from the venous blood of the two adult patients and cultured amniotic fluid fibroblasts. The 14 exons of PAX6 gene were amplified by polymerase chain reaction (PCR) using primers described by Redeker, et al. [10]. Obtained PCR products were sequenced bi-directionally using the ABI BigDye Terminator Cycle Sequencing kit v3.1 (ABI Applied Biosystems, Foster City, CA), according to the standard protocol.

\section{SNP-array assay}

Human cyto12 SNP-array (Illumina, USA) comprising around 300,000 SNPs was applied for whole genome scan on the two affected individuals (the proband II-1 and her brother II-3). SNP-array tests were performed according to the manufacturer's protocol (Illumina, USA), chromosome karyotype analysis was carried out by KaryoStudio V 1.3.11 (Illumina, USA) and GenomeStudio V2011.1 (Illumina, USA) respectively. We use hg18 genome coordinate in this study.

\section{Multiplex ligation-dependent probe amplification (MLPA) technique}

SALSA MLPA Kit P219-B2 PAX6 (MRC-Holland Amsterdam, Netherlands) was employed to verify the abnormal SNP-array findings and to test the DNA of the present fetus. The kit contains probes targeting each exon of PAX6 with the exception of exon 7 and 13, and multiple other genes within $11 \mathrm{p} 13$ region including BDNF, FSHB, DCDC1, ELP4, RCN1, WT1, LOC645981, LOC646008, HIPK3, LMO2, EHF, CD44 and SOX2. In brief, $100 \mathrm{ng}$ DNA samples were denatured for $5 \mathrm{~min}$ at $98^{\circ} \mathrm{C}$ and then cooled to $25^{\circ} \mathrm{C}$. Probes were mixed and hybridized with DNA samples at $60^{\circ} \mathrm{C}$ overnight and were then reacted with ligase 65 at $54^{\circ} \mathrm{C}$ for $15 \mathrm{~min}$, followed by $5 \mathrm{~min}$ at $98^{\circ} \mathrm{C}$ and kept at $4^{\circ} \mathrm{C}$. Finally, all probes and sample ligations were amplified by PCR using specific carboxyfluorescein (FAM) labeled PCR primers. Electrophoresis was performed using an $\mathrm{ABI}$ PRISM 3130 (Applied Biosystems) and Data was analyzed by GeneMarker V4.0 software. A peak area ratio between 0.7 and 1.3 times was considered as normal one, below 0.7 represents deletions and above 1.3 represents duplications. Each result was confirmed by two independent tests.

\section{Consent}

Written informed consent was obtained from the patient's parents for publication of this paper. A copy of the written consent is available for review by the Editor-in-Chief of this journal. This research was approved by the Ethics Committee of Nanjing Maternity and Child Health Care Hospital.

\section{Competing interests}

The authors declare that they have no competing interests.

\section{Authors' contributions}

$\mathrm{PH}, \mathrm{LM}, \mathrm{DM}$ carried out the molecular genetic studies. LY, ZX and drafted the manuscript. FQ, YW, JZ reviewed all the clinical data and genetic counseling. All authors read and approved the final manuscript. 


\section{Acknowledgements}

We are grateful to the patients and their families for their participation in this study. The study is supported by the National Natural Science Foundation of China (81300495), National Natural Science Foundation of Jiangsu Province (BK20141076), Key Technology R\&D Program of Jiangsu Province (BL2012039), and the Foundation of Jiangsu Provincial Department of Health (F201314, H201343).

\section{Author details}

'State key Laboratory of Reproductive Medicine, Department of Prenatal Diagnosis, Nanjing Maternity and Child Health Care Hospital Affiliated to Nanjing Medical University, 123\# Tianfei Street, Baixia District, Nanjing 210029, China. ${ }^{2}$ Department of Pathology, Nanjing University Medical School, Nanjing, China.

Received: 1 September 2014 Accepted: 9 January 2015

Published online: 22 January 2015

\section{References}

1. Blanco-Kelly F, Villaverde-Montero C, Lorda-Sanchez I, Millan JM, Trujillo-Tiebas MJ, Ayuso C. Guidelines for genetic study of aniridia. Arch Soc Esp Oftalmol. 2013;88:145-52.

2. Hingorani M, Hanson I, van Heyningen V. Aniridia. Eur J Hum Genet 2012;20:1011-7

3. Simpson TI, Price DJ. Pax6; a pleiotropic player in development. Bioessays. 2002;24:1041-51.

4. van Heyningen V, Williamson K. PAX6 in sensory development. Hum Mol Genet. 2002;11:1161-7.

5. Vincent M, Pujo A, Olivier D, Calvas P. Screening for PAX6 gene mutations is consistent with haploinsufficiency as the main mechanism leading to various ocular defects. Screening for PAX6 gene mutations is consistent with haploinsufficiency as the main mechanism leading to various ocular defects 2003;11:163-69.

6. Bamiou D, Musiek F, Sisodiya S, Free S, Davies R, Moore A, et al. Deficient auditory interhemispheric transfer in patients with PAX6 mutations. Ann Neurol. 2004;56:503-9.

7. Ahlqvist E, Turrini F, Lang S, Taneera J, Zhou Y, Almgren P, et al. A common variant upstream of the PAX6 gene influences islet function in man. Diabetologia. 2011;55:94-104.

8. Mitchell T, Free S, Williamson K, Stevens J, Churchill A, Hanson I, et al. Polymicrogyria and absence of pineal gland due to PAX6 mutation. Ann Neurol. 2003:53:658-63.

9. Malandrini A, Mari F, Palmeri S, Gambelli S, Berti G, Bruttini M, et al. PAX6 mutation in a family with aniridia, congenital ptosis, and mental retardation. Clin Genet. 2001;60:151-4.

10. Maekawa M, Iwayama Y, Nakamura K, Sato M, Toyota T, Ohnishi T, et al. A novel missense mutation (Leu46Val) of PAX6 found in an autistic patient. Neurosci Lett. 2009;462:267-71.

11. Dansault A, David G, Schwartz C, Jaliffa C, Vieira V, de la Houssaye G, et al. Three new PAX6 mutations including one causing an unusual ophthalmic phenotype associated with neurodevelopmental abnormalities. Mol Vis. 2007:13:511-23.

12. Drechsler M, Royer-Pokora B. A LINE element is present at the site of a $300-\mathrm{kb}$ deletion starting in intron 10 of the PAX6 gene in a case of familial aniridia. Hum Genet. 1996;98:297-303.

13. Crolla J, Cawdery J, Oley C, Young I, Gray J, Fantes J, et al. A FISH approach to defining the extent and possible clinical significance of deletions at the WAGR locus. J Med Genet. 1997;34:207-12.

14. Lauderdale J, Wilensky J, Oliver E, Walton D, Glaser T. 3' deletions cause aniridia by preventing PAX6 gene expression. Proc Natl Acad Sci U S A. 2000;97:13755-9

15. Davis L, Meyer K, Rudd D, Librant A, Epping E, Sheffield V, et al. Pax6 3 deletion results in aniridia, autism and mental retardation. Hum Genet. 2008:123:371-8.

16. Redeker E, de Visser A, Bergen A, Mannens M. Multiplex ligation-dependent probe amplification (MLPA) enhances the molecular diagnosis of aniridia and related disorders. Mol Vis. 2008;14:836-40.

17. Bayrakli F, Guney I, Bayri Y, Ercan-Sencicek A, Ceyhan D, Cankaya T, et al. A novel heterozygous deletion within the $3^{\prime}$ region of the PAX6 gene causing isolated aniridia in a large family group. J Clin Neurosci. 2009;16:1610-4.
18. Cheng F, Song W, Kang Y, Yu S, Yuan H. A 556 kb deletion in the downstream region of the PAX6 gene causes familial aniridia and other eye anomalies in a Chinese family. Mol Vis. 2011;17:448-55.

19. Zhang X, Zhang Q, Tong Y, Dai H, Zhao X, Bai F, et al. Large novel deletions detected in Chinese families with aniridia: correlation between genotype and phenotype. Mol Vis. 2011;17:548-57.

20. Wawrocka A, Sikora A, Kuszel L, Krawczynski MR. 11 p13 deletions can be more frequent than the PAX6 gene point mutations in Polish patients with aniridia. J Appl Genet. 2013;54:345-51.

21. Osumi N. The role of Pax6 in brain patterning. Tohoku J Exp Med. 2001;193:163-74.

22. Gérard M, Abitbol M, Delezoide A, Dufier J, Mallet J, Vekemans M. PAX-genes expression during human embryonic development, a preliminary report. C R Acad Sci III. 1995;318:57-66.

23. Bamiou D, Free S, Sisodiya S, Chong W, Musiek F, Williamson K, et al, Auditory interhemispheric transfer deficits, hearing difficulties, and brain magnetic resonance imaging abnormalities in children with congenital aniridia due to PAX6 mutations. Arch Pediatr Adolesc Med. 2007;165:463-9.

24. Cooper S, Hanson I. A screen for proteins that interact with PAX6: C-terminal mutations disrupt interaction with HOMER3, DNCL1 and TRIM11. BMC Genet. 2005:6:43.

25. Sisodiya S, Free S, Williamson K, Mitchell T, Willis C, Stevens J, et al. PAX6 haploinsufficiency causes cerebral malformation and olfactory dysfunction in humans. Nat Genet. 2001;28:214-6.

26. Ellison-Wright Z, Heyman I, Frampton I, Rubia K, Chitnis X, Ellison-Wright I, et al. Heterozygous PAX6 mutation, adult brain structure and fronto-striato-thalamic function in a human family. Eur J Neurosci. 2004;19:1505-12.

27. Kleinjan DA, Seawright A, Schedl A, Quinlan RA, Danes S, van Heyningen V. Aniridia-associated translocations, DNase hypersensitivity, sequence comparison and transgenic analysis redefine the functional domain of PAX6. Hum Mol Genet. 2001;10:2049-59.

28. Kleinjan DA, Seawright A, Elgar G, van Heyningen V. Characterization of a novel gene adjacent to PAX6, revealing synteny conservation with functional significance. Mamm Genome. 2002;13:102-7.

29. Gasten AC, Ramdas WD, Broer L, van Koolwijk LM, Ikram MK, de Jong PT, et al. A genetic epidemiologic study of candidate genes involved in the optic nerve head morphology. Invest Ophthalmol Vis Sci. 2012;53:1485-91.

\section{Submit your next manuscript to BioMed Central and take full advantage of:}

- Convenient online submission

- Thorough peer review

- No space constraints or color figure charges

- Immediate publication on acceptance

- Inclusion in PubMed, CAS, Scopus and Google Scholar

- Research which is freely available for redistribution 